\title{
MODELING ONE-DIMENSIONAL INCOMPRESSIBLE DUCT FLOWS
}

\author{
Jorge Luis Baliño \\ Escola Politécnica, Universidade de São Paulo \\ Av. Prof. Mello Moraes, 2231, CEP 05508-900, Cidade Universitária, São Paulo, SP, Brazil \\ E-mail: jlbalino@usp.br
}

\begin{abstract}
In this paper a Bond Graph methodology described in a previous contribution is used to model incompressible one-dimensional duct flows with rigid walls. Since the volumetric flow is independent of position, the balance equations can be simplified. The volumetric flow and a nodal vector of entropy are defined as Bond Graph state variables. The state equations and the coupling with the inertial and entropy ports are modeled with true bond graph elements.
\end{abstract}

Keywords- Bond Graphs, incompressible flow, one-dimensional duct flow, Finite Elements.

\section{INTRODUCTION}

The Bond Graph formalism allows for a systematic approach for representing and analyzing dynamic lumpedparameter systems $[7,12]$. Dynamic systems belonging to different fields of knowledge like Electrodynamics, Solid Mechanics or Fluid Mechanics can be described in terms of a finite number of variables and basic elements.

Fluid Dynamics is a defiant area for bondgraphers, because these systems are rigorously described by non-linear partial differential equations (PDEs) with important spatial effects and exhibit couplings between different energy domains.

In order to solve multidimensional problems with the aid of computer programs, it is important that these models can be implemented numerically. This task, main concern of the area of Computational Fluid Dynamics (CFD), is performed by systematically discretizing the continua, that is, by replacing the continuous variables by a combination of a finite set of nodal values and interpolating functions.

The first attempt to apply Bond Graphs in fluid dynamic systems with a systematic spatial discretization of flow fields, typical of CFD problems, appeared in [9]. A compressible flow was considered, although the formulation was restricted to prescribed shape functions and nodalization. Besides, heat conduction (which leads to convectiondiffusion problems) was not modeled.

In [1] a theoretical development of a general Bond Graph approach for CFD was presented. Density, entropy per unit volume and velocity were used as discretized variables for single-phase, single component flows. Timedependent nodal values and interpolation functions were introduced to represent the flow field. Nodal vectors of mass, entropy and velocity were defined as Bond Graph state variables. It was shown that the system total energy can be represented as a three-port $I C$-field. The conservation of linear momentum for the nodal velocity is represented at the inertial port, while mass and entropy conservation equations are represented at the capacitive ports. All kind of boundary conditions are handled consistently and can be represented as generalized modulated sources. The methodology was successful in different applications: one-dimensional convection-diffusion [10], one-dimensional problems with constant piecewise shape functions [2,5], shock tube problem [11] and single-phase, multicomponent flows [3,4].

In [6] the methodology was applied to model multidimensional incompressible flows. The distinctive characteristic of these flows is the role of pressure, which doesn't behave as a state variable but as a function that must act in such a way that the resulting velocity field has divergence zero. The system of equations for the momentum equation and for the incompressibility restriction is coincident with the one obtained by using the Galerkin formulation of the problem in the Finite Element Method, while for the thermal energy equation a Petrov-Galerkin formulation is used. The integral incompressibility restriction was derived based on the integral conservation of mechanical energy. All kind of boundary conditions are handled consistently and can be represented as generalized effort or flow sources for the velocity and entropy balance equations. A procedure for causality assignment was derived for the resulting graph, satisfying the Second principle of Thermodynamics.

The motivation of this paper is the application of the methodology described in [6] to incompressible onedimensional duct flows.

\section{TOTAL ENERGY PER UNIT VOLUME}

Let us consider the geometry of Fig. 1. For a onedimensional incompressible flow, the density $\rho_{0}$ is constant and the volumetric flow $Q$ is independent of position. The velocity $V$ can be readily determined as:

$$
V(x, t)=\frac{Q(t)}{A(x)}
$$

where $x$ is the axial position, $t$ is the time and $A$ is the passage area. The internal energy per unit volume $u_{v}\left(u_{v}=\right.$ $\rho_{0} \hat{u}$, where $\hat{u}$ is the internal energy per unit mass) is only a function of the entropy per unit volume $s_{v}\left(s_{v}=\rho_{0} \hat{s}\right.$, where $\hat{s}$ is the entropy per unit mass). The total energy per unit volume $e_{v}$ can be written as:

$$
e_{v}=u_{v}\left(s_{v}\right)+t_{v}^{*}(Q) \quad ; \quad t_{v}^{*}=\frac{1}{2} \rho_{0} \frac{Q^{2}}{A^{2}}
$$

where $t_{v}^{*}$ is the kinetic coenergy per unit volume. The following potentials are defined:

$$
p_{v}=\frac{d t_{v}^{*}}{d Q}=\rho_{0} \frac{Q}{A^{2}} \quad ; \quad \theta=\frac{d u_{v}}{d s_{v}}
$$


where $p_{v}$ and $\theta$ are correspondingly the pressure momentum per unit volume and the absolute temperature. For an incompressible fluid, the temperature can be expressed as:

$$
\theta=\theta_{R} \exp \left(\frac{s_{v}}{\rho_{0} c_{v}}\right)
$$

where $\theta_{R}$ is a reference temperature (for which the entropy is zero) and $c_{v}$ is the constant volume specific heat. The time derivative of (2) can be written as:

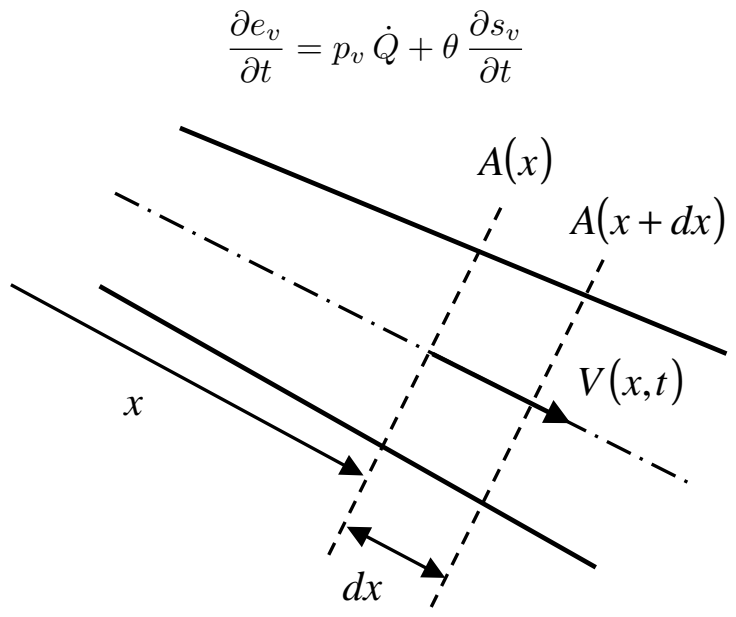

Fig. 1. Control volume for a one-dimensional incompressible duct flow.

Since the internal energy per unit volume is only a function of the entropy per unit volume, and the kinetic coenergy per unit volume is only a function of the volumetric flow, these two energy types can be split.

\section{CONSERVATION AND BALANCE EQUATIONS}

Neglecting normal viscous stresses and considering equal to unity the shape factors weighting the fluxes (momentum or kinetic energy) across the passage area, the linear momentum and thermal energy conservation equations for one-dimensional incompressible flows are:

$$
\begin{gathered}
\frac{\rho_{0}}{A} \dot{Q}=-\frac{\partial P}{\partial x}+\rho_{0} G_{x}-\frac{\rho_{0} Q^{2}}{A} \frac{d}{d x}\left(\frac{1}{A}\right)-\tau_{w x} \frac{\mathcal{P}_{w}}{A} \\
\theta \frac{\partial s_{v}}{\partial t}=-q_{w} \frac{\mathcal{P}_{h}}{A}-\frac{1}{A} \frac{\partial}{\partial x}\left(q_{x} A\right)-\frac{Q}{A} \frac{\partial}{\partial x}\left(\theta s_{v}\right) \\
+\rho_{0} \Phi+\frac{Q}{A} s_{v} \frac{\partial \theta}{\partial x}+\tau_{w x} Q \frac{\mathcal{P}_{w}}{A^{2}}
\end{gathered}
$$

where $P$ is the pressure, $\tau_{w x}$ is the viscous wall shear stress, $\mathcal{P}_{w}$ and $\mathcal{P}_{h}$ are correspondingly the wetted and heated perimeters, $G_{x}$ is the force per unit mass, $q_{w}$ and $q_{x}$ are correspondingly the wall and axial heat flux and $\Phi$ is the heat source per unit mass. The wall shear stress and heat flux can be modeled as:

$$
\tau_{w x}=\frac{1}{8} f \rho_{0} \frac{Q|Q|}{A^{2}} \quad ; \quad q_{w}=-H\left(\theta_{w}-\theta\right)
$$

where $f$ is the Darcy friction factor and $H$ is the heat transfer coefficient, being these two parameters obtained from suitable correlations. According to Fourier's law, the axial heat flux can be written as:

$$
q_{x}=-\lambda \frac{\partial \theta}{\partial x}=-\frac{\lambda \theta}{\rho_{0} c_{v}} \frac{\partial s_{v}}{\partial x}
$$

where $\mu$ and $\lambda$ are correspondingly the fluid viscosity and thermal conductivity. Multiplying (6) times the velocity, we obtain the mechanical energy conservation equation per unit volume:

$$
\begin{gathered}
p_{v} \dot{Q}=-\frac{Q}{A} \frac{\partial P}{\partial x}+\rho_{0} G_{x} \frac{Q}{A} \\
-\frac{\rho_{0} Q^{3}}{A^{2}} \frac{d}{d x}\left(\frac{1}{A}\right)-\tau_{w x} Q \frac{\mathcal{P}_{w}}{A^{2}}
\end{gathered}
$$

The balance equations (7) and (10) are power equations (per unit volume) corresponding to each one of the terms that contributes to the time derivative of the total energy per unit volume, namely (5). The balance equations show the power structure of the system. The coupling term $\tau_{w x} Q \frac{\mathcal{P}_{w}}{A^{2}} \geq 0$ represents the power transfer (mechanical energy dissipation) between the volumetric flow and entropy equations; this coupling term appears, with opposite signs, in the balance equations. Finally, the remaining terms are regarded as power sources.

It is important to notice that, since we started from the PDEs describing the dynamics, all the effects compatible with the one-dimensional approximation are modeled. A further simplification made in all bond graph modeling approaches is to neglect axial heat conduction, which is valid for advection dominated (high Peclet number) flows; this approximation has consequences in the causality assignment, as it will be seen in Section VIII.

The conservation and balance equations obtained in this Section can be used for turbulent flows considering the independent variables as mean values, since axial turbulent stresses and axial turbulent heat flux are not important. Turbulence effects are introduced through the friction factor and the heat transfer coefficient.

\section{DISCRETIZATION}

\section{A. Nodal entropy vector}

The description of the entropy per unit volume in the domain $\Omega$ is made in terms of a finite set of nodal values and interpolation functions, as in the Finite Element Method [8]:

$$
s_{v}(x, t)=\sum_{l=1}^{n} s_{v l}(t) \varphi_{S l}(x)=\underline{s}^{T} \cdot \underline{\varphi_{S}}
$$

where $\underline{s}_{v}($ size $n)$ is a time-dependent nodal vector, while $\underline{\varphi_{S}}$ is the corresponding nodal vector of interpolation or shape functions. Volume integration of any function $\psi$ in the domain $\Omega$ is defined as:

$$
\int_{\Omega} \psi d \Omega=\int_{0}^{L} A \psi d x
$$


where $L$ is the duct length. The nodal vector of entropy is defined as:

$\underline{S}=\underline{\underline{\Omega_{S}}} \cdot \underline{s_{v}} ;\left(\Omega_{S}\right)_{l j}=\Omega_{S l} \delta_{l j} ; \Omega_{S l}=\int_{0}^{L} A \varphi_{S l} d x$

where $\Omega_{S}$ is a diagonal volume matrix associated to the entropy per unit volume and $\delta_{l j}$ is the Kronecker's delta ( $\delta_{l j}=1$ if $l=j, \delta_{l j}=0$ otherwise). The system entropy can be obtained as:

$$
S=\int_{0}^{L} A s_{v} d x=\sum_{l=1}^{n} S_{l}
$$

\section{B. Total energy}

The system total energy $E$ is defined as the sum of the internal energy $U$ and the kinetic coenergy $T^{*}$ :

$$
E=U(\underline{S})+T^{*}(Q)
$$

where:

$E=\int_{0}^{L} A e_{v} d x ; U=\int_{0}^{L} A u_{v} d x ; T^{*}=\int_{0}^{L} A t_{v}^{*} d x$

From (16), it can be easily shown that the system kinetic coenergy can be expressed as:

$$
T^{*}=\frac{1}{2} I Q^{2} \quad ; \quad I=\rho_{0} \int_{0}^{L} \frac{d x}{A}
$$

where $I$ is the system hydraulic inertia. We define the following potentials:

$$
\begin{gathered}
p=\frac{d T^{*}}{d Q}=I Q=\int_{0}^{L} A p_{v} d x \\
\underline{\Theta}(\underline{S})=\frac{d U}{d \underline{S}}=\underline{\underline{\Omega_{S}}}{ }^{-1} \cdot\left[\int_{0}^{L} A \theta \underline{\varphi_{S}} d x\right]
\end{gathered}
$$

where $p$ is the system pressure momentum and $\underline{\Theta}$ is the nodal vector of temperature. Since the hydraulic inertia is constant, kinetic energy storage can be represented by an inertia $(I)$ relating the volumetric flow and pressure momentum, eq. (18), with generalized effort given by $I \dot{Q}$. On the other hand, internal energy storage can be represented by a capacitive $(C)$ multibond field.

The time derivative of (15) can be written as:

$$
\dot{E}=p \dot{Q}+\underline{\Theta}^{T} \cdot \underline{\dot{S}}
$$

It can also be shown that the volume integrals of the left side terms of (7) and (10) can be calculated as:

$$
\int_{0}^{L} A \theta \frac{\partial s_{v}}{\partial t} d x=\underline{\Theta}^{T} \cdot \underline{\dot{S}} ; \int_{0}^{L} A p_{v} \dot{Q} d x=p \dot{Q}
$$

\section{STATE EQUATIONS}

A. Inertial port

Integrating in position (10) and considering constant $G_{x}$, Bernoulli equation can be readily obtained as:

$$
I \frac{d Q}{d t}=\Delta P+\Delta P_{G}+\Delta P_{B}-\Delta P_{V W}
$$

where:

$$
\begin{gathered}
\Delta P=P_{0}-P_{L} \\
\Delta P_{G}=\rho_{0} G_{x} L \\
\Delta P_{B}=\frac{1}{2} \rho_{0} Q^{2}\left(\frac{1}{A_{0}^{2}}-\frac{1}{A_{L}^{2}}\right) \\
\Delta P_{V W}=\int_{0}^{L} \tau_{w x} \frac{\mathcal{P}_{w}}{A} d x
\end{gathered}
$$

In Eq. (22) to (26) it can be recognized the transient, gravitational, convective and frictional contributions to the pressure drop. It can be easily shown that the product of any pressure drop in (22) times $Q$ is equal to the corresponding term of (10) integrated in volume.

\section{B. Entropy Port}

Nodal entropy weight functions $w_{S l}(x, t)$ are introduced, as it is done in the Petrov-Galerkin formulation for the Finite Element Method [8]. The nodal entropy weight functions are introduced to satisfy the power interchanged by the system through the boundary conditions, as well as to share the importance of different power terms among neighboring nodes. These functions can be used, for instance, to introduce upwind schemes in convectiondiffusion problems $[2,5,10]$.

Each term of the entropy balance equation (7) is multiplied by $w_{S l}$. Then, the resulting terms are integrated over the domain $\Omega$ and Gauss' theorem is applied whenever necessary, obtaining:

$$
\underline{\dot{S}}=\underline{\dot{S}_{Q}^{(\Gamma)}}+\underline{\dot{S}_{C}^{(\Gamma)}}+\underline{\dot{S}_{Q W}}+\underline{\dot{S}_{Q}}+\underline{\dot{S}_{C}}+\underline{\dot{S}_{F}}+\underline{\dot{S}_{A}}+\underline{\dot{S}_{V W}}
$$

where:

$$
\begin{aligned}
& \dot{S}_{Q}^{(\Gamma)}=-\underline{\underline{\Theta}}^{-1} \cdot\left(q_{x L} A_{L} \underline{\delta_{l n}}-q_{x 0} A_{0} \underline{\delta_{l 1}}\right) \\
& \underline{\dot{S}_{C}^{(\Gamma)}}=-\underline{\underline{\Theta}}^{-1} \cdot\left[Q\left(\theta_{L} s_{v L} \underline{\delta_{l n}}-\theta_{0} s_{v 0} \underline{\delta_{l 1}}\right)\right] \\
& \underline{\dot{S}_{Q W}}=-\underline{\underline{\Theta}}^{-1} \cdot\left[\int_{0}^{L} q_{w} \mathcal{P}_{h} \underline{w_{S}} d x\right] \\
& \underline{\dot{S}_{Q}}=\underline{\underline{\Theta}}^{-1} \cdot\left[\int_{0}^{L} A q_{x} \frac{\partial \underline{w}_{S}}{\partial x} d x\right] \\
& \underline{\dot{S}}_{C}=\underline{\underline{\Theta}}^{-1} \cdot\left[Q \int_{0}^{L} \theta s_{v} \frac{\partial w_{S}}{\partial x} d x\right] \\
& \underline{\dot{S}_{F}}=\underline{\underline{\Theta}}^{-1} \cdot\left[\rho_{0} \int_{0}^{L} \Phi A \underline{w_{S}} d x\right]
\end{aligned}
$$




$$
\begin{gathered}
\underline{\dot{S}_{A}}=\underline{\underline{\Theta}}^{-1} \cdot\left[Q \int_{0}^{L} s_{v} \frac{\partial \theta}{\partial x} \underline{w_{S}} d x\right] \\
\dot{S}_{V W}=\underline{\underline{\Theta}}^{-1} \cdot\left[Q \int_{0}^{L} \tau_{w x} \frac{\mathcal{P}_{w}}{A} \underline{w_{S}} d x\right]
\end{gathered}
$$

In (28) to (35) the temperature matrix $\underline{\underline{\Theta}}$ results:

$$
(\Theta)_{l j}=\frac{1}{\Omega_{S j}} \int_{0}^{L} A \theta w_{S l} \varphi_{S j} d x
$$

The nodal vector of temperature is related to the temperature matrix as:

$$
\Theta_{j}=\sum_{l=1}^{n}(\Theta)_{l j}
$$

Taking into account (37) it can be verified that the product of the nodal vector of temperature times any nodal vector of entropy rate $\underline{\Theta}^{T} \cdot \dot{S}_{X}$ recovers the corresponding power of (7) integrated in the system volume. Thus, the products

$\underline{\Theta}^{T} \cdot \underline{\dot{S}}_{Q}^{(\Gamma)}$ and $\underline{\Theta}^{T} \cdot \underline{\dot{S}}_{C}^{(\Gamma)}$ recover correspondingly the power due to heat flux and entropy advection at the system boundary, while $\underline{\Theta}^{T} \cdot \dot{S}_{Q}$ and $\underline{\Theta}^{T} \cdot \underline{\dot{S}_{C}}$ are a power terms that

vanish, because $\sum_{l=1}^{n} w_{S l}=1$.

\section{DISSIPATION COUPLING}

It is necessary to represent the power coupling due to viscous dissipation (appearing in the balance equations per unit volume shown in Section III) to a discretized level. This representation relates generalized variables whose product gives rise to the dissipation power term, appearing in the velocity and entropy ports as:

$$
\underline{\Theta}^{T} \cdot \underline{\dot{S}_{V}}=\Delta P_{V} Q=Q \int_{0}^{L} \tau_{w x} \frac{\mathcal{P}_{w}}{A} d x
$$

The friction pressure drop can be expressed as:

$$
\Delta P_{V W}=\sum_{l=1}^{n} \Delta P_{V W l} ; \Delta P_{V W l}=\int_{0}^{L} \tau_{w x} \frac{\mathcal{P}_{w}}{A} w_{S l} d x
$$

In this way, we define a nodal vector of friction pressure drop as:

$$
\underline{\Delta P_{V W}}=\int_{0}^{L} \tau_{w x} \frac{\mathcal{P}_{w}}{A} \underline{w_{S}} d x
$$

From (35) and (40) we have:

$$
\underline{\dot{S}_{V W}}=\left(\underline{\underline{\Theta}}^{-1} \cdot \underline{M_{V W}}\right) Q ; \underline{\Delta P_{V W}}=\left(\underline{\underline{\Theta}}^{-1} \cdot \underline{M_{V W}}\right)^{T} \cdot \underline{\Theta}
$$

where $M_{V W}$ is a nodal vector, defined as:

$$
\underline{M_{V W}}=\int_{0}^{L} \tau_{w x} \frac{\mathcal{P}_{w}}{A} \underline{w_{S}} d x
$$

Equation (41) defines a multibond transformer modulated by the state variables, in which $\underline{\underline{\Theta}}^{-1} \cdot M_{V W}$ is the dissipation coupling vector. Besides satisfying conservation of energy through the transformer, namely (38), it will be seen in Section IX that the resulting causality also satisfies the Second Principle of Thermodynamics.

\section{SYSTEM BOND GRAPH}

The system Bond Graph is shown in Fig. 2. Energy storage (kinetic and internal) are represented correspondingly by an inertia $(I)$ and a capacitive $(C)$ field. At the 1 -junction with common $Q$ we add all the nodal pressure drops; in this way, the effort balance represents Bernoulli equation in terms of the volumetric flow. At the 0 -junction with common $\underline{\Theta}$ we add all the nodal entropy rates; in this way, the flow balance represents the thermal energy conservation equation for the nodal entropy values.

The resulting Bond Graph is similar to the one obtained in [12] for fluid dynamic systems, but there are slight differences. Since the bond with the convective pressure drop $\Delta P_{B}$ may supply or extract power, we prefer to model it as an effort source. Besides, pressure drop due to friction $\Delta P_{V W}$ are decomposed and coupled to the entropy port through the modulated transformer connecting the 1 and 0 junctions, obtaining the entropy rate due to viscous dissipation.

The sources $S$ (the ones connected to the bonds with $P_{0}$, $P_{L}, \quad \underline{\dot{S}_{Q}^{(\Gamma)}}$ and $\underline{\dot{S}_{C}^{(\Gamma)}}$ ) represent different source terms related to the boundary conditions; in each single port these sources behave as effort or flow sources, depending on the boundary conditions.

The rest of the sources, effort $S_{e}$ or flow $S_{f}$ represent volumetric power terms; the determination of causality for these sources and for the bonds connected to the modulated transformer $M T F$ results from the standard causality extension procedure detailed in Section IX. As we saw before, the net power input (sum over the bonds) corresponding to the multibonds with the entropy rate ${\underline{\dot{S}_{Q}}}_{\text {and }} \underline{\dot{S}_{C}}$ are zero.

\section{INITIAL AND BOUNDARY CONDITIONS}

Initial conditions can be written as:

$$
Q(t=0)=Q_{0} \quad ; \quad \underline{S}(t=0)=\underline{S_{0}}
$$

The boundary conditions establish relationships among the variables corresponding to the nodes located at the system surface and can be regarded (in the Bond Graph methodology) as the input variables. It is necessary, for the model being mathematically well defined, that the boundary conditions allow to determine univoquely the causality for all the elements in the resulting Bond Graph.

For a one-dimensional geometry, the boundary conditions are established at $x=0$ and/or $x=L$. Possible boundary conditions in an incompressible duct flow for the inertial port are $\Delta P(t)$ or $Q(t)$.

If axial heat conduction is taken into account (advectiondifussion flows), the balance equation is second order and we need two boundary conditions for the entropy port, which can be $\theta(0, t)$ or $q_{x}(0, t)$ and $\theta(L, t)$ or $q_{x}(L, t)$. For advection dominated flows, axial heat conduction is neglected and the resulting balance equation is first order, 

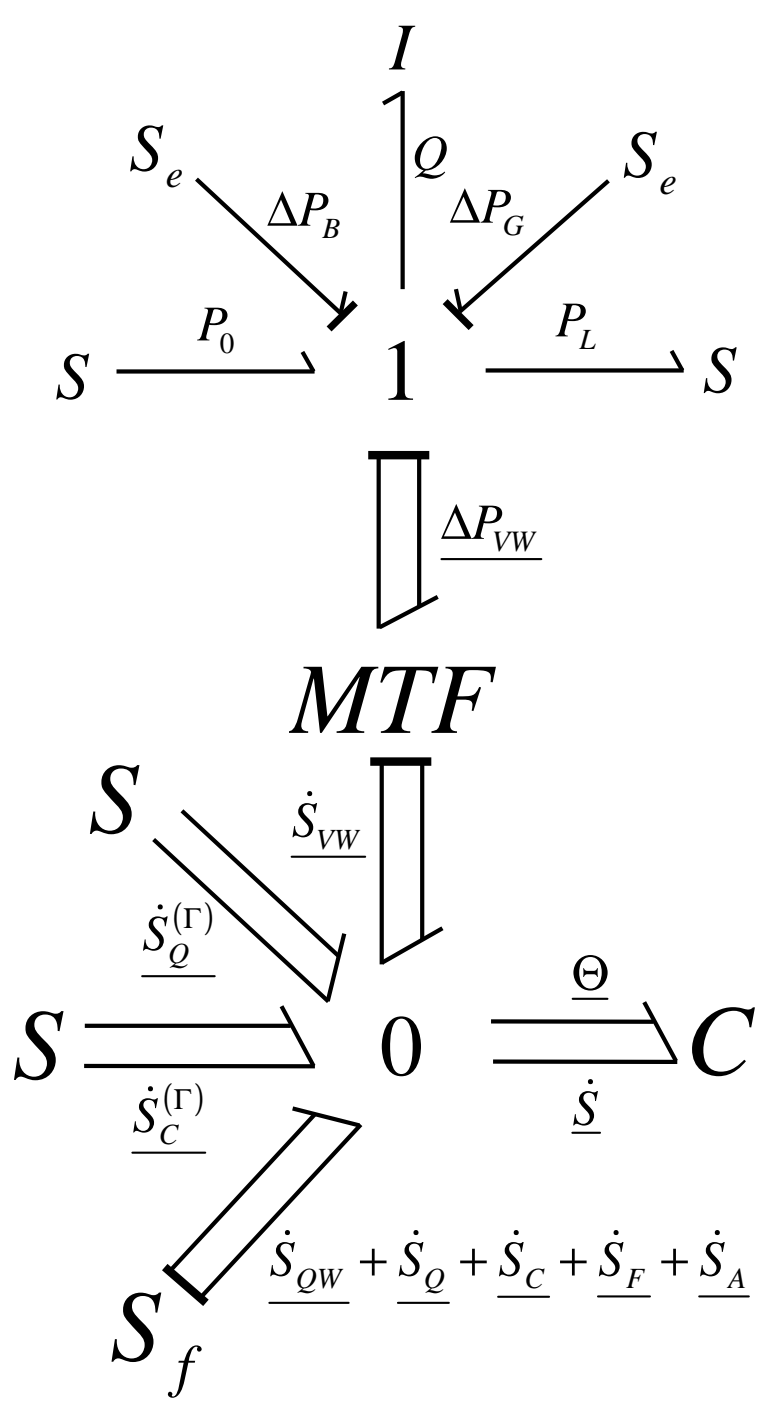

Fig. 2. System Bond Graph for a one-dimensional incompressible duct flow.

needing only one temperature as boundary condition. For any case, $\theta_{w}(x, t)$ or $q_{w}(x, t)$ must be specified.

\section{CAUSALITY ASSIGNMENT}

In [6], the sequencial causal assignment procedure described in [12] was applied. Sources are chosen first, the required causality is assigned and the causal implications are extended through the graph as far as possible, using the constraint elements (in this case 0 -junctions, 1 -junctions and transformers). Then, the ports corresponding to the storage elements (in this case inertia and $C$-field) are chosen, integral causality is assigned and, again, the causal implications are extended through the graph as far as possible. Regarding causality extension through the $M T F$ element, the constitutive laws are sets of linear relationships among the variables involved. Thus, causality can be extended for a bond with a variable only when the bonds corresponding to the rest of the variables in the linear relationship have assigned causalities.

It is worth noting that, since the interpolation and weight functions are zero at the boundaries 0 and $L, \dot{S}_{Q l}^{(\Gamma)}=0$ at the bonds corresponding to inner nodes, as indicated in (28).

For advection-diffusion flows, entropy rate or temperature are imposed at the 0-junctions by the bonds with $\dot{S}_{Q 1}^{(\Gamma)}$ or $\dot{S}_{Q n}^{(\Gamma)}$; in this case, the sources connected to the bonds with $\dot{S}_{C l}^{(\Gamma)}$ always result flow sources. For advection flows, $\dot{S}_{Q l}^{(\Gamma)}=\dot{S}_{Q l}=0$ and temperature can be imposed at the 0-junctions by the bonds with $\dot{S}_{C 1}^{(\Gamma)}$ or $\dot{S}_{C n}^{(\Gamma)}$.

As a consequence of the causality extension, the sources connected to the bonds with $\Delta P_{G}$ and $\Delta P_{B}$ always behave as effort sources, and the sources connected to the bonds with $\dot{S}_{Q W}, \dot{S}_{Q}, \underline{\dot{S}_{C}}, \underline{\dot{S}_{F}}$ and

$\dot{S}_{A}$ always behave as flow sources. Besides, causality for the bonds connected to the modulated transformer (the ones with $\Delta P_{V W}$ and $\dot{S}_{V}$ ) is also defined, always resulting $Q$ an input to the transformer and $\dot{S}_{V}$ an output; this indicates that fluid motion generates the irreversible entropy rate, in agreement with the Second Principle of Thermodynamics. Besides, $\underline{\Theta}$ always results an input to the transformer and $\Delta P_{V W}$ an output, indicating that temperature influences the viscous force through the temperature dependence of viscosity.

\section{CONCLUSIONS}

In this paper a Bond Graph methodology described in a previous contribution was used to model incompressible one-dimensional duct flows with rigid walls. Since the volumetric flow is independent of position, the momentum equation could be simplified and lumped. The volumetric flow and a nodal vector of entropy were defined as Bond Graph state variables. The state equations and the coupling with the inertial and entropy ports were modeled with true bond graph elements. This contribution shows that starting from the governing PDEs equations and using discretization techniques coming from CFD is the right strategy for producing general models, framed within the Bond Graph theory, for Fluid Dynamic Systems.

\section{ACKNOWLEDGMENTS}

The author wishes to thank "Conselho Nacional de Desenvolvimento Científico e Tecnológico" (CNPq, Brazil) for the financial support as Scientific Productivity Scholar.

\section{REFERENCES}

[1] Baliño, J. L., Larreteguy, A. E. \& Gandolfo, E. F., A General Bond Graph Approach for Computational Fluid Dynamics. Part I: Theory, 2001 International Conference on Bond Graph Modeling and Simulation (ICBGM'2001), The Society for Computer Simulation, pp. 41-46. ISBN 1-56555-221-0, 2001. 
[2] Baliño, J. L., Bond-Graph Approach for Computational Fluid Dynamics: a Comparison with other Numerical Methods, Second IEEE International Conference on System, Man and Cybernetics (SMC'02), Paper TA1B3 (in CD, IEEE No. 02CH37349C, ISBN 0-7803-7438-X), 6 p., 2002.

[3] Baliño, J. L., BG-CFD Methodology for Multicomponent Solutions. Part I: Multivelocity Model, 2003 International Conference on Bond Graph Modeling and Simulation (ICBGM'2003), The Society for Computer Simulation, pp. 41-46, 2003.

[4] Baliño, J. L., BG-CFD Methodology for Multicomponent Solutions. Part II: Diffusion Model, 2003 International Conference on Bond Graph Modeling and Simulation (ICBGM'2003), The Society for Computer Simulation, pp. 47-52, 2003.

[5] Baliño, J. L., Bond-Graph Formulation of CFD Problems with Constant Piecewise Shape Functions, International Journal of Heat and Technology, vol. 21, 1, pp. 59-66, 2003.

[6] Baliño, J. L., Bond Graph Modeling of Incompressible Flows, 17th. IMACS World Congress, Scientific Computation, Applied Mathematics and Simulation (IMACS 2005), en CD, ISBN 2915913-02-1, 8 p., 2005.

[7] Borutzky, W., Bond Graphs. A Methodology for Modelling Multidisciplinary Dynamic Systems, SCS Publishing House, ISBN 3-936150-33-8, 2004.

[8] Cuvelier, C., Segal, A. \& van Steenhoven, A. A., Finite Element Methods and Navier-Stokes Equations, D. Reidel Publishing Company, Holland, 1986.

[9] Fahrenthold, E. P. \& Venkataraman, M., Eulerian Bond Graphs for Fluid Continuum Dynamics Modeling, ASME Journal of Dynamic Systems, Measurement, and Control, vol. 118, pp. 48-57, 1996.
[10] Gandolfo, E. F., Larreteguy, A. E. \& Baliño, J. L., A General Bond Graph Approach for Computational Fluid Dynamics. Part II: Applications, 2001 International Conference on Bond Graph Modeling and Simulation (ICBGM'2001), The Society for Computer Simulation, pp. 47-52. ISBN 1-56555-221-0, 2001.

[11] Gandolfo Raso, E. F., Larreteguy, A. E. \& Baliño, J. L., Bond-Graph Modeling of 1-D Compressible Flows, Second IEEE International Conference on System, Man and Cybernetics (SMC'02), Paper TA1B4 (in CD, IEEE No. 02CH37349C, ISBN 0-7803-7438-X), 6 p., 2002.

[12] Karnopp, D. C., Margolis, D. L. \& Rosenberg, R. C., System Dynamics. Modeling and Simulation of Mechatronic System, 3d Ed., Wiley Interscience, ISBN 0-471-33301-8, 2000.

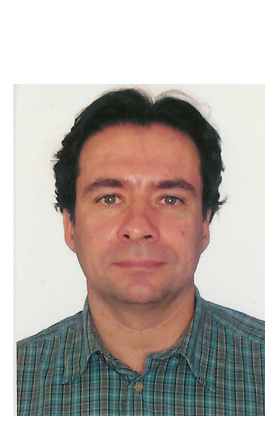

\section{About the author}

Jorge Luis Baliño was born in Buenos Aires, Argentina. He graduated as nuclear engineer (1983) and made a Ph.D. in Nuclear Engineering (1991) at Instituto Balseiro (Argentina). He worked for Techint S.A. (1983-1984) and Centro Atómico Bariloche and Instituto Balseiro (1985-2000) in Argentina. He worked as Visiting Professor for Instituto de Pesquisas Energéticas e Nucleares (2001-2003) at São Paulo, Brazil. Since 2004 he works as Professor at Universidade de São Paulo. His research interests are Fluid Dynamics, Heat Transfer and Multiphase Flow. 